\title{
Mucositis oral en niños con cáncer y su relación con limitación de actividades
}

\author{
Miguel Ángel Palomo-Colli* ${ }^{*}$, Diana Carolina Soltero-Becerril2, Luis Enrique Juárez Villegas', \\ Carlo Cicero-Oneto ${ }^{1}$, José Félix Gaytán-Morales ${ }^{1}$, Iván Castorena-Villa ${ }^{1}$ y Mónica Mier-Cabrera ${ }^{3}$ \\ ${ }^{1}$ Departamento de Oncología; ${ }^{2}$ Departamento de Pediatría Médica. Hospital Infantil de México Federico Gómez, Ciudad de México; ${ }^{3}$ Departamento \\ de Oncología, Hospital Regional de Alta especialidad de Ixtapaluca, Ixtapaluca. México
}

\section{Resumen}

Introducción: La mucositis oral (MO) es un efecto adverso común del tratamiento con quimioterapia y radioterapia en pacientes pediátricos con cáncer, la complicación puede observarse durante el tratamiento oncológico y es importante debido a que afecta directamente a este. Métodos: Evaluamos y seguimos 402 niños durante dos semanas después del inicio de la quimioterapia en el periodo comprendido entre junio del 2012 y junio del 2016. Los niños completaron un cuestionario diario con preguntas relacionadas a la MO desde el inicio de la quimioterapia (día 0), hasta el día 14. Resultados: El 73\% $(n=307)$ de los pacientes desarrollaron MO. La mayoría de los casos fueron de grado 2 y 3. De las 307 evaluaciones, el $12 \%(n=32)$ fueron grado 1 y el $49 \%(n=205)$, grado 2. La media de tiempo de inicio de la MO fue de 4.5 (DE: 2.5; rango: 2-9; IC 95\%: 3.8-5.3) días después del inicio de la quimioterapia, con una media de duración de 6.5 (desviación estándar [DE]: 4; rango: 2-13; intervalo de confianza [IC] 95\%: 5.2-9.4) días con pico al día 7.1 (DE 2.8; rango: 4-11; IC 95\%: 6.8-8.4). Conclusiones: $L a M O$ se asocia con efectos negativos en las necesidades básicas de los niños con cáncer.

Palabras clave: Mucositis. Cáncer. Quimioterapia. Calidad de vida. Niños

\section{Oral mucositis in children with cancer and their relationship with limitation of activities}

\begin{abstract}
Introduction: Oral mucositis (OM) is a common chemo and radiotherapy adverse effect in oncological pediatric patients, oral complications may be observed during chemotherapy and are important side effects that may directly affect the anticancer treatment. Methods: We included 420 children and monitored them for 2 weeks after the initiation of chemotherapy between June 2012 and June 2016. Subjects were asked to use a daily diary to complete the related questions of the OM Daily Questionnaire from the start of chemotherapy to day 14. Results: Overall, $73 \%(n=307)$ of paediatric patients developed OM. Most cases were Grade 2 and 3. Of these, $12 \%(n=32)$ were Grade 1 , and 49\% ( $n=205)$ Grade 2. The mean time to onset of OM was 4.5 (standard deviation [SD]: 2.5; range: 2-9; 95\% confidence interval [CI]: 3.8-5.3) days after the start of chemotherapy, with a mean duration of 6.5 (SD: 4; range: 2-13; 95\% Cl: 5.2-9.4) days and with the peak at day 7.1 (SD: 2.8; range: 4-11; 95\% Cl: 6.8-8.4). Conclusions: OM is associated with negative effects on clinical outcomes.
\end{abstract}

Key words: Mucositis. Cancer. Chemotherapy. Quality of life, Children.

\section{Correspondencia:}

${ }^{*}$ M.A. Palomo-Colli

E-mail: phalomi@gmail.com

DOI: 10.24875/j.gamo.M18000137

1665-9201/@ 2018 Sociedad Mexicana de Oncología. Publicado por Permanyer México SA de CV. Este es un artículo Open Access bajo la licencia CC BY-NC-ND (http://creativecommons.org/licenses/by-nc-nd/4.0/).

Fecha de recepción: 15-01-2017

Fecha de aceptación: 14-02-2017

Gac Mex Oncol. 2018;17:87-92 www.gamo-smeo.com
a licencia CC 


\section{Introduccion}

La MO se refiere a las lesiones eritematosas y ulcerosas de la mucosa oral observadas en pacientes con cáncer tratados con quimioterapia y/o con radioterapia en campos que implican la cavidad oral. Este término, tal como describe Köstler ${ }^{1}$, surgió a finales de los años 80 para definir la inflamación en la cavidad oral inducida por la quimioterapia y la radioterapia, pues esta inflamación representaba una entidad distinta a otras lesiones orales, denominadas estomatitis, y tenía diferente etiopatogenia. Sin embargo, según otros autores, la mucositis consiste en una inflamación de las mucosas que conduce a ulceración y rotura de su integridad, y puede afectar a todo el tracto gastrointestinal, desde la boca hasta el ano?

La MO es la inflamación que tiene lugar en el epitelio oral, a consecuencia de los tratamientos antineoplásicos como la radioterapia, la quimioterapia o el trasplante de células progenitoras hematopoyéticas, siendo muy frecuente durante el tratamiento de padecimientos oncohematológicos.

Se estima que hasta el $50 \%$ de los pacientes que reciben quimioterapia para tumores sólidos o linfomas desarrolla MO o gastrointestinal.

Se estima que la mucositis se presenta en el $22 \%$ de los casos en mucosa oral, en el $8 \%$ en mucosa oral y gastrointestinal, y en el $7 \%$ en la gastrointestinal de los pacientes tratados con quimioterapia; sin embargo, en pacientes que reciben quimioterapia a dosis altas como parte de regímenes para el trasplante de células progenitoras hematopoyéticas puede llegar a presentarse hasta en el $75-80 \%$ de los pacientes ${ }^{3,4}$.

La lesión de la barrera mucosa es una complicación que muchos niños con enfermedad oncológica presentan durante su tratamiento. La lesión de la barrera mucosa puede ser muy dolorosa para los pacientes y ponerlos en alto riesgo de desarrollar complicaciones. Cuando la barrera de las mucosas se rompe, el paciente está en riesgo de desarrollar una infección grave que puede amenazar la vida. Los pacientes que reciben quimioterapia a menudo desarrollan neutropenia, que implica un riesgo mayor de infecciones si se agrega al daño a las mucosas.

La capacidad de reconocer a pacientes con alto riesgo de tener lesiones de la barrera mucosa puede detener la progresión de la lesión y permite dar un tratamiento temprano que evite complicaciones adicionales.

La MO es un evento secundario frecuente que deteriora la calidad de vida del paciente, agrava las condiciones clínicas, aumenta el riesgo de infección y puede llevar a un retraso en los tiempos de administración de los esquemas de quimioterapia, lo cual lleva a una intensidad de dosis no idónea, lo cual finalmente puede repercutir en el pronóstico del niño con cáncer. La MO es la segunda causa de toxicidad limitante de dosis en pacientes que reciben quimioterapia. Además, puede ser la causa de ingresos hospitalarios, prolongar la estancia hospitalaria y en ocasiones predispone al ingreso a unidades de cuidados intensivos.

La incidencia de complicaciones orales puede reducirse llevando a cabo evaluaciones de la cavidad oral antes de iniciar la terapia, eliminando las fuentes potenciales de infección o irritación y usando medidas preventivas durante todo el tratamiento.

Los factores de riesgo para desarrollar mucositis clásicamente están asociados con la modalidad, la intensidad y la vía de administración de los fármacos antineoplásicos.

La combinación de la terapia puede incrementar la severidad de la mucositis.

Se ha demostrado que los niños en tratamiento antineoplásico presentan más posibilidades de mucositis debido a la tasa alta de recambio celular, al igual que en personas mayores de 50 años; otros factores adicionales son: cavidad oral mal cuidada, patología dental y prótesis defectuosas, que deberán ser solucionados antes del inicio de la terapia. El grado y la severidad de la mucositis en el paciente con cáncer está determinado por el tipo de fármaco empleado, la dosis empleada y por la susceptibilidad genética del paciente ${ }^{5-7}$.

\section{Valoración de la mucositis oral}

En los últimos 30 años se han desarrollado distintos modelos para documentar y cuantificar los cambios en los tejidos epiteliales de la cavidad oral y las alteraciones de su función, durante y después del tratamiento del cáncer. En la actualidad existen una gran variedad de escalas para valorar la extensión y severidad de la MO, útiles en la práctica clínica y como recurso en investigación?.

La Organización Mundial de la salud (OMS) define el grado de las lesiones de la mucositis según su severidad (Tabla 1). Es una escala simple y fácil de usar en la práctica clínica, que combina parámetros tanto objetivos como subjetivos para valorar el grado de lesión de la mucosa oral.

\section{Impacto económico de la mucositis}

Los pacientes que padecen un grado significativo de MO requieren de medidas de apoyo, como el uso de 
Tabla 1. Escala de toxicidad oral de la OMS

\begin{tabular}{|l|l|}
\hline Grado & Manifestaciones \\
\hline 0 & Sin evidencia subjetiva u objetiva de mucositis \\
\hline 1 & Dolor oral con o sin eritema, sin úlceras \\
\hline 2 & Eritema y ulceración, puede comer sólidos \\
\hline 3 & Eritema y ulceración, solo tolera dieta líquida \\
\hline 4 & Eritema y ulceración, no puede alimentarse \\
\hline
\end{tabular}

nutrición parenteral total, restitución hídrica y profilaxis antibiótica. Esto puede elevar sustancialmente el costo del tratamiento.

En un estudio realizado en el Hospital Universitario de Connecticut, el costo estimado por hospitalización fue de $\$ 3,893$ USD por ciclo de quimioterapia sin mucositis, $\$ 6,277$ USD por ciclo de quimioterapia y con MO y $\$ 9,132$ USD por ciclo de quimioterapia y con mucositis, tanto oral como gastrointestinal ${ }^{8}$.

Se encontró que cada grado de aumento de la severidad de la mucositis se asocia con un día adicional de fiebre, un aumento del doble del riesgo de infección grave, 2.7 días adicionales de nutrición parenteral total, 2.6 días más de tratamiento analgésico, un aumento de 2.6 días en la estancia intrahospitalaria y un incremento de 3.7 en el riesgo de mortalidad por cada 100 días de estancia intrahospitalaria, lo que contribuye colectivamente a un costo adicional de $\$ 25,000$ USD al costo de la hospitalización?

\section{Material Y métodos}

Este estudio prospectivo de cohorte observacional fue realizado de junio del 2012 a junio del 2016, en el Departamento de Oncología del Hospital Infantil de México Federico Gómez. Se eligió a niños de tres años o más que estuvieran en tratamiento con quimioterapia para cualquier tipo de neoplasia, que desearan participar en el estudio (así como sus cuidadores primarios) y tuviesen la capacidad de leer y responder el cuestionario diario de la MO pediátrica adaptado (OMDQ) versión final ${ }^{10}$.Esto se realizó solo en los pacientes que ingresaban en el área de quimioterapia de corta estancia.

Se empleó la escala de la OMS para establecer la MO y sus grados (Tabla 1).

La aplicación del instrumento (OMDQ) fue explicada por médicos pediatras y oncólogos a los cuidadores y los niños, durante la estancia en las salas de quimioterapia de corta estancia (hospitalización), requiriendo un lapso de 10-15 minutos. Los encuestados contestaron el cuestionario durante 14 días, iniciando 24 horas después del inicio del esquema de quimioterapia y las respuestas se registraron al término de las 24 horas de la fecha calendario.

Con respecto al cuestionario diario de la OMDQ, se evaluó la presencia de dolor en boca y garganta cada 24 horas empleando símbolos faciales incorporados en la versión autocompletada del niño que fueron adaptados de la escala de puntuación del dolor FACES de Wong-Baker. La escala de caras ha demostrado fiabilidad y validez en el uso del dolor del paciente de autoinforme en niños ${ }^{11}$.

Del diario de MO (OMDQ) se evaluaron cuatro preguntas en forma separada: 1) intensidad de dolor en boca y garganta, 2) impacto de la mucositis en el comer, 3) en el beber y 4) en el hablar.

Un médico examinó la cavidad bucal de los niños cuando estos referían presencia de dolor para determinar el estado de la cavidad bucal y decidir el tipo de tratamiento que se requería. Todos los niños que presentaron mucositis fueron evaluados y tratados de acuerdo con la evolución que presentaran (ambulatoria u hospitalaria).

\section{Resultados}

El total de quimioterapias administradas en el área de hospitalización en el periodo del estudio fue de 4,215 infusiones y se incluyeron en el estudio 420 niños que aceptaron participar. Según el género, 240 fueron niños $(57 \%)$ y $180(43 \%)$ niñas. Los fármacos que se administraron fueron: arabinósido de citosina (28\%), metotrexato a dosis altas ( $2 \mathrm{~g} / \mathrm{m}^{2}$ o más) (35\%), antraciclinas (37\%), etopósido (14\%), vincristina (12\%), ciclofosfamida $(9 \%)$ y otros $(13 \%)$.

Utilizando la escala de valoración de mucositis de la OMS se encontró que, de los 420 pacientes incluidos, 113 pacientes (27\%) no tuvieron MO, 51 niños (12\%) cursaron con mucositis grado 1, 205 niños (49\%) con grado 2, $46(11 \%)$ con grado 3 y cinco niños (1\%) con grado 4 . El porcentaje de pacientes que presentaron MO en todo el grupo de estudio fue del $73 \%(n=307)$ (Fig. 1).

El tiempo medio para el desarrollo de la MO fue de 4.5 días (DE: 2.5; rango: 2- 9: IC 95\%: 3.8-5.3) días después del inicio de la quimioterapia, con una media de duración de 6.5 días (DE: 4; rango: 2-13; IC 95\%: 5.2- 9.4) con un pico al día 7.1 (DE: 2.8; rango: 4-11; IC 95\%: 6.8-8.4). 


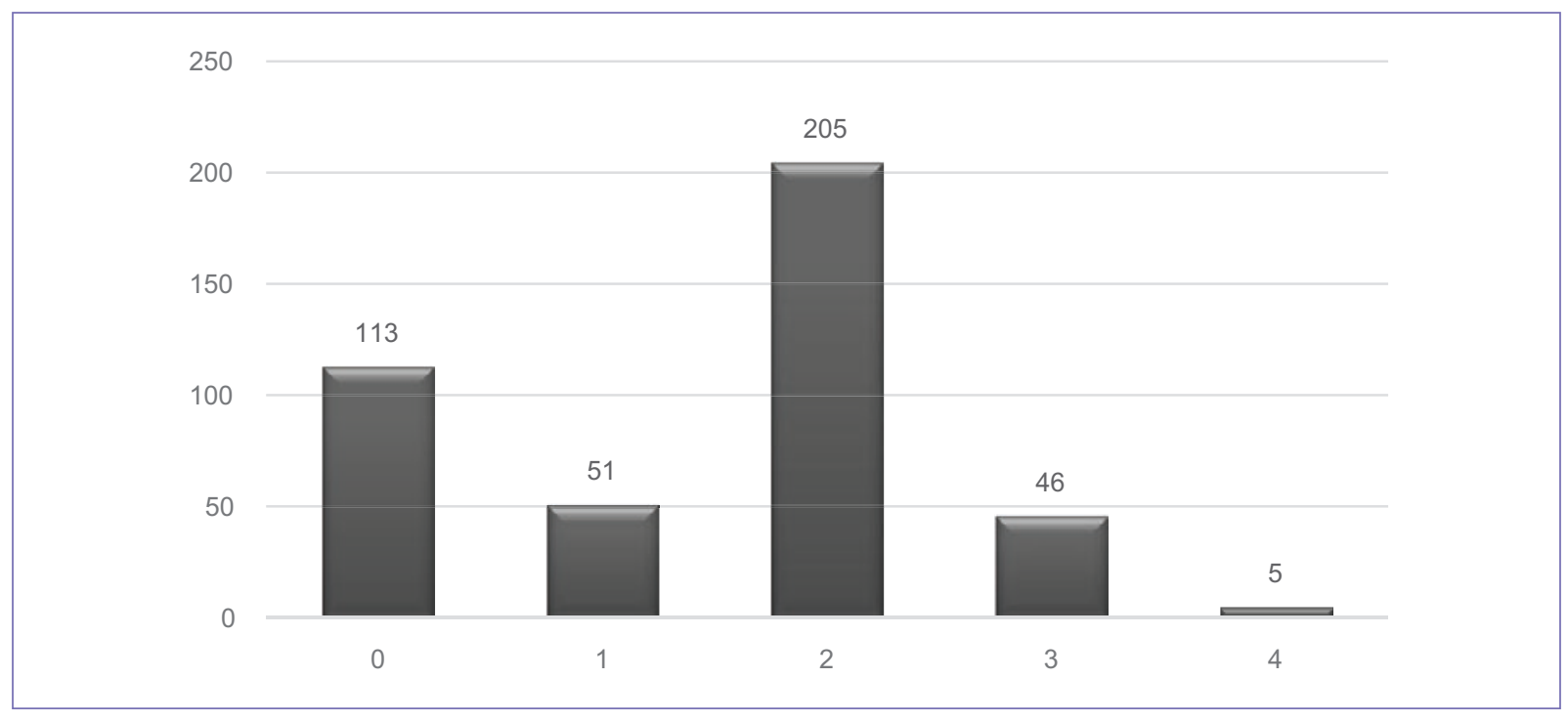

Figura 1. Grados de mucositis según la OMS.

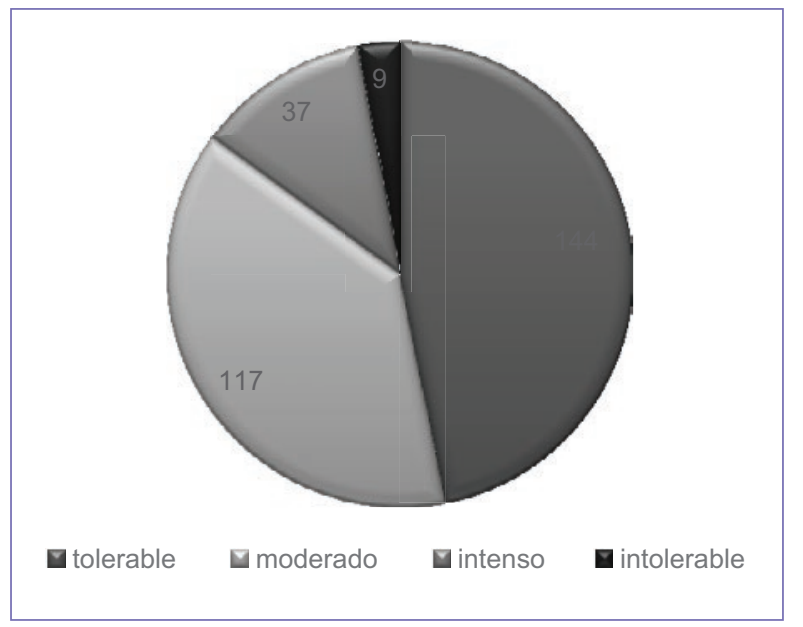

Figura 2. Dolor en niños con mucositis.

Las lesiones se describieron de acuerdo con la escala de evaluación de la MO (OMAS) 7,9 . De los 307 pacientes que presentaron algún grado de mucositis, el $14 \%$ tenían solo eritema de la mucosa, el $51 \%$ tenían eritema y la presencia de una úlcera de menos de $1 \mathrm{~cm}$, el $23 \%$ presentaban lesiones ulcerativas mayores de $1 \mathrm{~cm}$, el restante $10 \%$ presentaba úlceras confluentes mayores de $3 \mathrm{~cm}$ y el $2 \%$ presentaron necrosis y sangrado de las lesiones.

En los pacientes con diferentes grados de mucositis ( $n=307$ ) se encontró que el $47 \%$ refirió dolor tolerable, el $38 \%$ dolor moderado, el $12 \%$ dolor intenso y el $3 \%$ dolor intolerable (Fig. 2).
Los hallazgos presentes durante los episodios de mucositis con respecto a la ingesta de alimentos fueron los siguientes: el $41 \%$ toleró dieta sólida, al $46 \%$ se le dificultó deglutir sólidos y se les ofreció dieta líquida, y el restante $13 \%$ no toleró ninguna de las dos dietas y se tuvo que mantener en ayuno hasta la remisión del cuadro con líquidos intravenosos y/o nutrición parenteral de acuerdo al tiempo en el cual se controlaba el dolor (Fig. 3).

De los 141 niños que solo toleraron líquidos, el 43\% no tuvieron limitaciones para beber, el 33\% tenían limitada un poco la capacidad para beber y el $24 \%$ mostraba limitación para beber y presencia de sialorrea (Fig. 3).

Con respecto a la limitación para hablar en los niños con mucositis ( $n=307)$ : el $53 \%$ no tuvo ninguna limitación, el 24\% refería un poco de dolor al hablar, el $13 \%$ tuvo dolor moderado al hablar y el $10 \%$ eran incapaces de hablar por el dolor extremo (Fig. 3).

El tratamiento para la $\mathrm{MO}$ en la institución donde se realizó el estudio no tiene apego a alguna guía de práctica clínica, ya que, como se mencionó, estas son muy diversas. A los niños con mucositis grado 1 y grado 2 con ausencia de dolor o dolor tolerable se les ofreció dieta normal o blanda, aseo de la boca con agua carbonatada y enjuagues bucales con Gelclair ${ }^{\circledR}$ para proteger el área de las lesiones y analgésicos a requerimiento. En los niños con mucositis grado 2 o 3 con dolor moderado o intenso, se dio dieta blanda o líquida, enjuagues con líquidos fríos, enjuagues con Gelclair $^{\circledR}$ y analgesia por vía tópica y/o sistémica por 


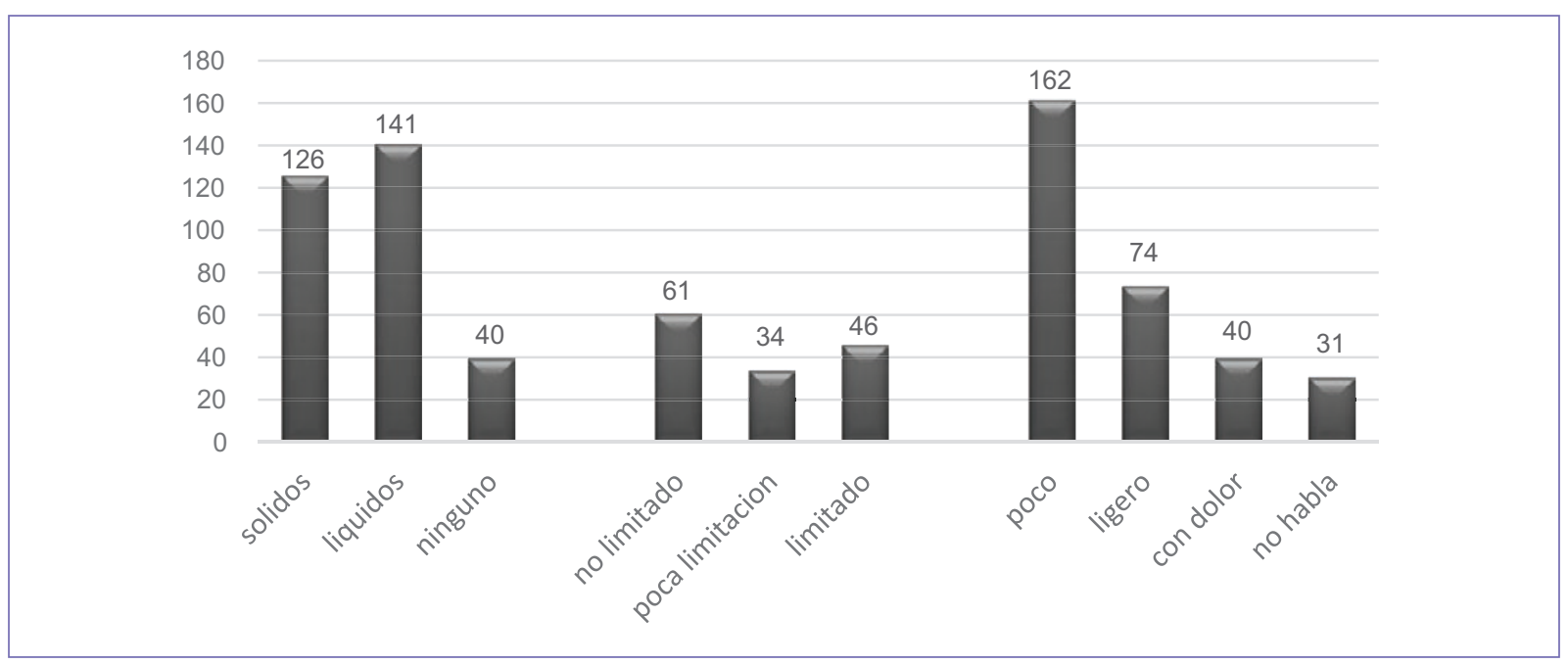

Figura 3. Limitaciones en las actividades en los niños con mucositis (deglutir, beber y hablar).

vía oral. Los niños con mucositis grado 304 y con dolor intolerable se trataron con ayuno, soluciones parenterales y/o nutrición parenteral total a requerimiento, enjuagues con Gelclair ${ }^{\circledR}$ y analgésicos intravenosos a requerimiento.

Requirieron hospitalización 40 niños (13\%) debido a presencia de dolor intenso e intolerable, mucositis grado 3 y 4 o por asociación a neutropenia y fiebre. El tiempo medio de hospitalización fue de 6 días (DE. 2.5; rango: 3- 28; IC 95\%: 3.8-12.3), todos ellos requirieron de antibióticos sistémicos.

\section{Discusión}

La MO es un efecto adverso en los niños que reciben tratamiento. En nuestro estudio el porcentaje de presentación es alto (73\%), los grados 3 y 4 se presentaron en un 15 y un $1.6 \%$ respectivamente. El porcentaje de $\mathrm{MO}$ elevado es probablemente debido a sesgo de selección, ya que no todos los niños que requirieron de quimioterapia en el periodo evaluado aceptaron participar en el estudio y existe la probabilidad de que los niños que presentaron esta complicación fueran los que completaron la evaluación.

El dolor relacionado con la $\mathrm{MO}$ en este estudio condiciona una frecuencia considerable de limitaciones relacionadas con la función de la cavidad oral, reflejada en las limitaciones para la ingesta de sólidos y líquidos, y para hablar, comparado con el estudio de Ip, et al., en el cual se evalúan puntuaciones relacionadas con las limitaciones en actividades en niños (MTS, mouth and throat soreness score), se refiere que tiene un rango del 18 al 35\% relacionado con las actividades de comer, beber, hablar, deglutir y dormir, un $25 \%$ de la población del estudio tiene limitaciones relacionadas con todas las actividades evaluadas y el 39\% reportó al menos limitación en dos actividades. En la evaluación realizada en nuestra población, el dolor severo (intenso e intolerable), que tiene un impacto relacionado con las actividades básicas de comer y beber, se presentó en el $15 \%$ de la población; entre estos pacientes, el 10\% eran incapaces de hablar por el dolor secundario a la mucositis y el $25 \%$ tuvo una limitación severa para las actividades de comer, beber y hablar. A diferencia del estudio referido, nosotros no evaluamos la limitación para las funciones de dormir y deglutir.

En los pacientes que tienen puntuaciones altas en la evaluación de dolor en boca y garganta (MTS 3-4) existe una relación con pérdida de peso $\geq 2 \mathrm{~kg}$ y también con mayor empleo de terapia de líquidos intravenosos, uso de analgésicos y antibióticos sistémicos orales e intravenosos. No fue posible en nuestro grupo de estudio evaluar el impacto relacionado con el decremento ponderal, ya que no se determinó en forma sistematizada el registro del peso previo y al final de la resolución del evento de mucositis ${ }^{12}$.

Un aspecto a mencionar es que el número de hospitalizaciones en estudios relacionados con la mucositis es bajo o no se requiere, a diferencia de lo que ocurrió en el grupo que nosotros evaluamos, en el cual se requirió de hospitalización en el $13 \%$ de los niños debido a las comorbilidades asociadas. En este estudio no evaluamos los factores que se asociaban a la 
mucositis. Todos los niños con neutropenia fueron hospitalizados y tratados con antibióticos intravenosos.

Los niños con neutropenia tienen un riesgo incrementado de mucositis severa debido al bajo recuento de neutrófilos, esenciales para proteger la mucosa, y también puede afectar la capacidad de proliferación de las células del epitelio oral ${ }^{13}$. Además de incrementar el riesgo de colonizar, la mucosa oral dañada permite un aumento de las citocinas proinflamatorias, lo que favorece el agravamiento de la mucositis ${ }^{14}$. Hay otros factores, como los genéticos, que pueden ser predisponentes para el desarrollo de mucositis y constituyen una potencial área de estudio de interés.

\section{Conclusiones}

Consideramos que es necesario hacer seguimiento de todos los niños con cáncer que requieran de fármacos que produzcan daño a la mucosa y proporcionar tratamiento temprano con el objetivo de disminuir el número de hospitalizaciones relacionados con esta complicación. Así mismo, es necesario tratar todas las condiciones que favorecen el problema (caries, mala higiene bucal, deficiencia en las técnicas de aseo bucal, empleo de fármacos y sustancias que incrementen el riesgo). La evaluación de la calidad de vida en niños con cáncer debe ser parte fundamental para el tratamiento integral y la toma de decisiones en pro de mejorar la atención y disminuir los factores que pueden impactar en una falta de apego al tratamiento.

\section{Conflicto de intereses}

Los autores declaran no tener ningún conflicto de interés.

\section{Agradecimientos}

Nuestro agradecimiento especial a todos los niños y sus familias que dieron su tiempo a este estudio. También manifestamos nuestra gratitud a los estudiantes, médicos y personal de enfermería que participaron en diversos aspectos de este estudio.

\section{Bibliografía}

1. Köstler WJ, Hejna M, Wenzel C, Zielinski CC. Oral mucositis complicating chemotherapy and/or radiotherapy: options for prevention and treatment. CA Cancer J Clin. 2001;51:290-315.

2. McGuire DB. Mucosal tissue injury in cancer therapy. More than muscositis and mouthwash. Cancer Pract. 2002;10:179-91.

3. Elting LS, Cooksley C, Chambers M, Cantor SB, Manzullo E, Rubenstein EB. The burdens of cancer therapy. Clinical and economic outcomes of chemotherapy-induced mucositis. Cancer. 2003;98(7):1531-9.

4. Vera-Llonch M, Oster G, Ford CM, Lu J, Sonis S. Oral mucositis and outcomes of allogeneic hematopoietic stem-cell transplantation in patients with hematologic malignancies. Support Care Cancer. 2007; 15(5):491-6.

5. Balducci L, Exterrman M. Management of cancer in the older person: a practical approach. Oncologist. 2000;20:224-37.

6. Peterson DE, Cariello A. Mucosal damage: a major risk factor for severe complications after cytotoxic therapy. Sem Oncol. 2004;31:35-44.

7. Niscola P, Romani C, Cupelli L, Scaramucci L, Tendas A, Dentamaro T, et al. Mucositis in patients with hematologic malignancies: an overview. Haematologica. 2007;92:222-31.

8. Elting LS, Cooksley C, Chambers M, Cantor SB, Manzullo E, Rubenstein EB. The burdens of cancer therapy. Clinical and economic outcomes of chemotherapy-induced mucositis. Cancer. 2003;98(7):1531-9.

9. Sonis ST, Oster G, Fuchs H, Bellm L, Bradford WZ, Edelsberg J, et al. Oral mucositis and the clinical and economic outcomes of hematopoietic stem-cell transplantation. J Clin Oncol. 2001;19(8):2201-5.

10. Tomlinson D, Isitt J, Barron R, Doyle J, Judd P, Gassas A, et al. Determining the understandability and acceptability of an oral mucositis daily questionnaire. J Pediatr Oncol Nurs. 2008;25(2):107-11.

11. Wong D, Baker C. Pain in children: Comparison of assessment scales. Pediatr Nurs. 1998;14:9-17.

12. Ip WY, Epstein JB, Lee V, Yuen HL, Li R, Thompson DR, et al. Oral mucositis in paediatric patients after chemotherapy for cancer. Hong Kong Med J. 2014;20(Suppl 7):S4-8.

13. Witko-Sarsat V, Rieu $P$, Descamps-Latscha $B$, Lesavre $P$, Halbwachs-Mecarelli L. Neutrophils: molecules, functions and pathophysiological aspects. Lab Invest. 2000;80:61753.

14. Sonis ST, Etling LS, Keefe D, Peterson DE, Schubert M, Hauer-Jensen M, et al. Perspectives on cancer therapy-induced mucosal injury: pathogenesis, measurement, epidemiology, and consequences for patients. Cancer. 2004;100(9 Suppl):1995-2025. 